\title{
Summary Talk: Experimental High Energy Physics
}

\author{
J. C. Anjos \\ Centro Brasileiro de Pesquísas Físicas \\ Rua Dr. Xavier Sigaud 150, Rio de Janeiro, 22290-180, RJ, Brasil \\ e-mail: janjos@cbpf.br
}

Received on 25 April, 2001

\begin{abstract}
A summary of the contributions on Experimental High Energy Physics at the XXI Brazilian National Meeting on Particle and Fields is presented. There were a total of 28 contributions, 21 in Panel Sessions and 7 in Oral Sessions.
\end{abstract}

\section{Introduction}

The main impression left in the Sessions dedicated to Experimental High Energy Physics is that there is presently a lot of activity of Brazilian groups in ten different experiments. A qualitative change in the participation of the Brazilian groups working in International Collaborations was also observed. In the eighties most of the participation was in Data Analysis and manpower for mounting the experiments. In the late nineties we observe a growing leadership in hardware projects for detectors and front-end electronics. We present below the list of the experiments for which there were contributions at this meeting:

\footnotetext{
Research and Development (R\&D) Projects in 4 experiments:

ATLAS (Cern) - Higgs and Beyond...

LHCb (Cern) - CP violation in the B system.

AUGER OBSERVATORY (Argentine) - Extreme energies cosmic rays.
}

EASCAMP (Campinas) - Extensive air showers.

Physics Analysis Results from 4 experiments: E791 (Fermilab) - Charm Hadroproduction.

FOCUS (Fermilab) - Charm Photoproduction.

D0 (Fermilab) - $p \bar{p}$ collisions at $1.8 \mathrm{TeV}$.

DELPHI (Cern) $-\mathrm{e}^{+} \mathrm{e}^{-}$collisions at the $\mathrm{Z}$ mass and beyond.

Interesting experimental projects presented in other Sessions of the Meeting:

GRAVITON Project (São Paulo) - Detection of gravitational waves [1].

ATHENA's Project (Cern) - Production and study of antihydrogen [2].

We will now present the summary of the contri- butions for each experiment and the reference to the abstract in the Program of the Meeting, to these Proceedings or to already published papers. Experiment

\section{ATLAS:}

Five communications at the meeting. Main activities concentrated in calorimetry. A group from COPPE/UFRJ has developed front-end electronics for the read-out of the tile calorimeter. The system will sum the signals from different cells of the calorimeter for trigger validation of events. A prototype was built in Brazil and is being tested for future production [3].

The COPPE group has also developed a software algorithm based on neural networks for discrimination between electrons and jets for the second level trigger for ATLAS [4].

Alternative algorithms for discriminating between electrons and jets using a topologic mapping of the energy deposition in the calorimeter were also presented $[5],[6]$.

Experiment LHCb: Intense activity from UFRJ group: four presentations, two on hardware projects, one on muon trigger simulation and one on the estimate of signal level for the rare decay $B_{s}^{0} \rightarrow \mu^{+} \mu^{-}$.

1) Performance of Wire Pad Chambers:

Prototypes of wire Pad chambers that will be used in the muon system for LHCb were tested by the UFRJ group with minimum ionizing particles and the results were presented [7]. Part of the chambers will be built in Brazil by the UFRJ group.

\section{2) The CARIOCA chip:}

Prototype of integrated circuit has been developed with a very fast and low noise preamplifier. Low gain version was developed to be used on the wire PAD 
Chambers of the LHCb muon system [8].

\section{3) Software Development:}

Detector inefficiencies, dead time, noise and cross talk effects were included in the digitalization module of the LHCb simulation program [9].

4) Study of the LHCb sensitivity to the rare decay $B_{s}^{0} \rightarrow \mu^{+} \mu^{-}$.

Simulation study of UFRJ group shows that present design of the detector appears to be suitable for the clear observation of this rare decay [10].

Experiment AUGER: A detailed article about the

Auger Project is included elsewere in these Proceedings [11]. During 2001 the first stage of the project, the Engineering Array, covering an area of $54 \mathrm{~km} 2$, will be completed in Malargue, Argentina, site of the Southern Hemisphere Observatory.

The status of the project was presented. The Auger Progress Report of February 2001 indicates that 21 of the 40 Cerenkov tanks of the array are already deployed and the electronics of the tanks are being mounted. A major breakthrough was achieved: for the first time the Central Data Acquisition System has communicated with surface detector stations in the observatory site. The satellite internet connection from Malargue is now operational. The first monitoring data from the tanks are already in the Auger Web Server.

For the Engineering Array the ground detectors will be overlooked by two fluorescence detector telescopes. Following the Progress Report, the Los Leones fluorescence detector building is ready and the mirror support structure for the fluorescence telescope is already in place. The first optical filter was shipped to Malargue and the first full camera has been successfully tested in Rome and will soon be headed for the site.

The Rio group (CBPF/PUC/UFF) is responsible for the installation of the surface detectors and for the simulation and reconstruction software [12],[13],[14]. An engineer is also involved with the Central Data Acquisition System (CDAS) project.

The Campinas group (UNICAMP) is responsible for manufacturing and for shipping the Cerenkov tanks to Malargue and for the design and construction of part of the fluorescence detectors: diaphragm, shutters and correction lenses. The group is also involved with the simulation of extensive air showers using the generation code CORSIKA and using different models for the hadronic interactions simulated by SIBYLL and QGSJET [15].

Experiment EASCAMP: Data obtained during the period 1998-2000 with the central muon detector of EASCAMP (Campinas) was presented. [16].The Streamer Tubes, their operation mode and data acquisition system were described. A study of the performance and stability of the streamer chambers during the data acquisition period was shown. The zenithal distribution of muons obtained from the data was presented and compared with other experiments, like EASTOP (Gran Sasso, Italy).

Experiment E791: Final results from 4 analyses per-

formed recently by the CBPF group were presented. Although representing about $10 \%$ of the number of collaborators, the CBPF group was responsible for about $20 \%$ of the publications of this experiment.

1) Amplitude analysis of the decays of the $D^{+}$ and $D_{s}^{+}$in 3 pions:

A Dalitz Plot analysis was done with a sample of $1172 \pm 61 D^{+}$and $848 \pm 44 D_{s}^{+}$.

The amplitude analysis has shown a dominant contribution of $\pi \pi$ scalar resonances. It was possible to measure with very good precision the mass and width of several light $\pi \pi$ resonances. For the first time a clear signal of the scalar meson $\sigma(500)$ with mass $478 \pm 24 \pm 9$ $\mathrm{MeV}$ and width $324 \pm 41 \pm 7 \mathrm{MeV}$ was observed [17].

2) Particle-antiparticle production asymmetries for $\Lambda_{c}, \Lambda, \Xi$ and $\Omega$ baryons:

Particle-antiparticle asymmetries were measured as functions of Feynman $x_{F}$ (scaled longitudinal momentum) and transverse momentum $p_{T}^{2}$ in the ranges $-0.12<x_{F}<0.12$ and $0<p_{T}^{2}<4$ for the hyperons and $-0.1<x_{F}<0.6$ and $0<p_{T}^{2}<8$ for the $\Lambda_{c}$. No asymmetry is predicted by perturbative QCD. Measured asymmetry is then a consequence of the hadronization process, especially leading particle effects.

Substantial asymmetries were found for hyperons even at $x_{F}=0$. Leading particle effects observed in hyperon production are in qualitative agreement with theoretical predictions [18].

For the $\Lambda_{c}$ a constant asymmetry of $12 \%$ was observed [19].

\section{Experiment FOCUS: 1) Photoproduction asym-}

metries of the charm baryons $\Lambda_{c}, \Sigma_{c}^{++}$and $\Sigma_{c}^{0}$ :

As in hadroproduction no asymmetry is expected in perturbative QCD. The measurement of photoproduction asymmetries for these baryons could then provide valuable information on the hadronization process, which is one of the least understood phenomena in QCD.

Preliminary results on the charm-anticharm photoproduction asymmetry using $\Lambda_{c}^{+} / \Lambda_{c}^{-}, \Sigma_{c}^{++} / \Sigma_{c}^{--}$and $\Sigma_{c}^{0} / \bar{\Sigma}_{c}^{0}$ were presented [20]. The asymmetry was studied in intervals of $p_{T}^{2}, p_{L}$, and $x_{F}$ for the case of $\Lambda_{c}$ baryons, while global values were extracted for the $\Sigma_{c}$ baryons, due to the lack of statistics. 
As in hadroproduction, a constant asymmetry of $12 \%$ for the $\Lambda_{c}$ was observed. Very preliminary results indicate a higher asymmetry, of the order of $19 \%$, for the $\Sigma_{c}$ baryons.

2) Preliminary $\Lambda_{c}$ branching ratio measurements from FOCUS.

The large charm sample collected by FOCUS, over one million fully reconstructed charm events, allow high precision measurements of charm particle properties. In particular FOCUS has reconstructed over 20,000 $\Lambda_{c}$ baryons, the world largest sample.

The branching ratios of the decay modes $\Lambda_{c} \rightarrow$ $\Lambda \pi \pi \pi$ and $\Lambda_{c} \rightarrow p \bar{K}^{0}$ relative to the dominant decay channel $\Lambda_{c} \rightarrow p K \pi$ were presented [21]. The preliminary results are in agreement, within errors, with the values reported by the Particle Data Group. The study of sistematic errors and the branching ratio of 11 other $\Lambda_{c}$ decay modes are in progress.

Experiment D0: Diffractive Physics will be one of the topics investigated by the experiment D0 in the next collider run, starting in April 2001. To accomplish this goal the Brazilian group has proposed the construction of a new sub-system to be incorporated in the D0 spectrometer: the Forward Proton Detector. The Roman Pots, insertion devices in the beam line to house the new detectors, were built at the LNLS in Campinas and the detector design and simulation is under the responsibility of the Brazilian group (CBPF/UERJ/UFRJ/UFBa/IFT/LNLS).

1) Studies of the effect of multiple interactions on the diffractive events at Do.

The ocurrence of multiple interactions in the same beam crossing was simulated and its effect on triggering diffractive events was studied [22]. Time information provided by the luminosity monitors and the event multiplicity were used for helping efficiently trigger on difractive processes.

\section{2) Study of Hard Diffraction at D0.}

Hard diffraction results from the last D0 run were presented. Measurement of rates, rapidity gaps, transverse energy and energy dependence were presented and compared with predictions of several models [23]. The gap fraction of diffractive dijet events relative to all dijets was presented for forward jets $(0.65 \% \pm 0.04 \%)$ and for central jets $(0.22 \%+0.05 \%-0.04 \%)$ at $1.8 \mathrm{TeV}$.

\section{Experiment DELPHI: The CERN Large Electron}

Collider - LEP was shutdown in November 2000, after 12 years of intense study of $\mathrm{e}^{+} \mathrm{e}^{-}$collisions in the energy range 91 - $209 \mathrm{GeV}$. Very precise tests of the Standard Model were performed. At the meeting several analyses carried out by the Brazilian groups of UFRJ, CBPF and UERJ searching for new physics were presented, as well as a talk on the search for the Higgs boson:

\section{1) Higgs searches at LEP-II:}

This was one of the most awaited talks of the meeting since at the highest energies of LEP-II a few events were found compatible with Higgs bosons produced in the process $\mathrm{e}^{+} \mathrm{e}^{-} \rightarrow Z^{*} \rightarrow Z^{0} H^{0}$. A detailed article about the Higgs search at LEP-II is included elsewere in these Proceedings [24]. The Higgs search was done by the four LEP experiments and after applying very tight cuts to reduce the backgroud 3 events from ALEPH and one from L3 survived as Higgs candidates. In the combined data from the 4 LEP experiments an excess of events for Higgs masses near $114 \mathrm{GeV}$ was observed, $2.9 \sigma$ away from the expected background. Although not enough to confirm the Higgs discovery, this result allows one to set a limit for the Higgs mass at $95 \%$ Confidence Level at $m_{H}>113.2 \mathrm{GeV} / c^{2}$.

\section{2) Search for staus:}

Data collected in the energy range 192 to $202 \mathrm{GeV}$ were analysed looking for the staus, the supersymmetric partner of the taus in the context of the Minimal Supersymmetric Standard Model. The preliminary results obtained by the UFRJ group show an agreement with standard model spectations [25].

\section{3) Search for four charged leptons final} states:

The six final states of charged four-leptons produced in $\mathrm{e}^{+} \mathrm{e}^{-}$collisions in the energy range $184-202$ $\mathrm{GeV}$ were analysed. Deviations from the Standard Model cross-section predictions would be a signal for new physics. The total cross-section were calculated in a blind analysis and compared with the ones expected from simulations. Good agreement was found [26].

\section{4) Search for spontaneous R-parity violation} at LEP-II:

In the supersymmetric extensions of the Standard Model the discrete symmetry called R-parity plays a determinant role in the phenomenology of the models. In the case when this symmetry is not conserved the single production of supersymmetric particles can take place. Searches for spontaneus R-parity violating signals at LEP2 were performed under the assumption of R-parity violation in the third lepton family. The expected topology for the decay of a pair of charginos into two acoplanar taus plus missing energy showed no evidence for a signal in the DELPHI data [27]. 


\section{References}

[1] A Primeira Fase do Projeto Gráviton: o Detetor de Ondas Gravitacionais Mario Schenberg - Odylio D. Aguiar et al-oral presentation.

[2] Low energy (anti)atoms for precision tests of basic Physics - D. M. Silveira, O. Pereira, M. Veloso and Claudio L. Cesar - Braz. J. Phys., this issue, (2001).

[3] O Sistema Somador para Formar o Sinal de Trigger do Calorímetro Hadrônico do ATLAS - Augusto Santiago Cerqueira, José Manoel de Seixas, Luiz Pereira Calôba, Felipe Soares C. Rodrigues, Bruno de Carvalho Costa and Ricardo Rezende Ramos - panel presentation.

[4] Mapeamento em Anéis para uma Separação Neuronal Elétron-Jato usando Calorímetros Multi-camadas e Multi-segmentados - André Rabello dos Anjos and José Manoel de Seixas - panel presentation.

[5] Um Mapeamento Topológico para um Discriminador Neuronal de Partículas Baseado em Calorímetros Segmentados - Marcos da Rocha Vassali and José Manoel de Seixas - oral presentation.

[6] Novos Algoritmos Baseados na Transformada de Hough para a Detecção de Traços num Detector de Fibras Óticas Cintilantes - Augusto Cesar Heluy Dantas, José Manoel de Seixas and Pablo Tupinambá Campos - panel presentation.

[7] Performance of Wire Pad Chambers for the Muon System of the LHCb Experiment - Bernard M. Maréchal, Danielle Magalhães Moraes, Érica Polycarpo, Leandro Salazar de Paula and Miriam Gandelman - panel presentation.

[8] Fast binary Front-End implemented in a $0.25 \mu \mathrm{m}$ CMOS technology using a novel current-mode technique - Danielle Magalhães Moraes, F. Anghinolfi, P. Jarron, A. Rivetti and P. Devall - panel presentation.

[9] Simulation of the digitization in the $\mathrm{LHCb}$ monte carlo program and study of its influence on the L0 muon trigger performance - S. Amato, P. Colrain, J. R. T. de Mello Neto, M. Gandelman, J. H. Lopes - panel presentation.

[10] Study of the LHCb sensitivity to the rare decay $B_{s}^{0} \rightarrow$ $\mu^{+} \mu^{-}$- Bernard M. Maréchal, Érica Polycarpo and Miriam Gandelman - panel presentation.

[11] Cosmic Accelerators and Terrestrial Detectors - R. C. Shellard - Braz. J. Phys., this issue, (2001).

[12] Simulação das contribuições devidas à Luz Cerenkov e à Atenuação Atmosférica nos Detetores de Fluorescência do Observatório Pierre Auger - Marcia Gonçalves do Amaral - panel presentation.

[13] Estudo da Eficiência dos Detectores de Fluorescência do Observatório Pierre Auger na Reconstrução da Energia a partir dos Chuveiros de Raios Cósmicos em função de um teto de nuvens de altura variavel - Danays Morejón González, Ronald C. Shellard and Marcia Gonçalves do Amaral - panel presentation.

[14] Reconstrução Geométrica de Raios Cósmicos Altamente Energéticos no Observatório Pierre Auger - Johana Chirinos Díaz and Ronald Cintra Shellard - panel presentation.

[15] Caracterizaç ão de Chuveiros Atmosféricos - Fernando Catalani and José Augusto Chinellato - panel presentation.

[16] Múons Observados com o Detetor Central do EASCAMP - Anderson Campos Fauth and Silvia Paganini - panel presentation.

[17] Resultados Finais da Analise de Amplitudes dos Canais $D^{+}$e $D_{s} \rightarrow 3 \pi$ com dados do Experimento E791 (FERMILAB) - I. Bediaga, A. Massafferri, J. M. de Miranda and A. Reis - panel presentation.

[18] Studies in the Production of Baryons in $500 \mathrm{GeV} / \mathrm{c} \pi^{-}$ Nucleon Interactions - Javier Solano and J. C. Anjos panel presentation.

[19] $\Lambda_{c}^{+} \Lambda_{c}^{-}$asymetries in $500 \mathrm{GeV} / \mathrm{c} \pi^{-}-N$ interactions - J. C. Anjos, J. Magnin, F. R. A. Simão and J. Solano - panel presentation.

[20] Photoproduction Asymetries of the Charm Baryons $\Lambda_{c}$, $\Sigma_{c}^{++}$and $\Sigma_{c}^{o}$ at FERMILAB Experiment E831/FOCUS - João dos Anjos, Cecilia Uribe Estrada, Alberto Sánches Hernández and Marleigh Sheaff - panel presentation.

[21] Preliminary $\Lambda_{c}$ Branching Ratio Measurements from FOCUS - João C. dos Anjos and Eleazar Cuautle panel presentation.

[22] Studies of the Effect of Multiple Interactions on the Diffractive Events at the D0 Detector - Wagner de Paula Carvalho and Andre Sznajder - panel presentation.

[23] Hard Diffraction at D0 - Gilvan A. Alves - oral presentation.

[24] Searches at LEP2 - Marcia Begalli - Braz. J. Phys., this issue, (2001).

[25] Update on the search for staus in the context of the MSSM within the DELPHI collaboration - Sandra Amato, Miriam Gandelman, José Helder Lopes and Mikael Berggren - panel presentation.

[26] Charged four-leptons Production at LEP2 Energies in DELPHI - Marcia Begalli, Luiz Martins Mundim Filho and María Elena Pol - oral presentation.

[27] Search for Spontaneous $R$-parity violation at LEP2 Danielle Magalhães Moraes, Leandro Salazar de Paula and Miriam Gandelman - oral presentation. 